\title{
Small Intestine Hexose Transport in Experimental Diabetes Increased Transporter mRNA and Protein Expression in Enterocytes
}

\author{
Charles F. Burant, ${ }^{*}$ Susan Flink, ${ }^{\star}$ Alexander M. DePaoli, ${ }^{*}$ Janet Chen, ${ }^{\star}$ Wen-Sen Lee, ${ }^{5}$ \\ Matthias A. Hediger, ${ }^{5}$ John B. Buse, ${ }^{*}$ and Eugene B. Chang ${ }^{*}$ \\ Department of Medicine, Sections of ${ }^{*}$ Endocrinology and ${ }^{\ddagger}$ Gastroenterology, University of Chicago, Chicago, Illinois 60637; and \\ ${ }^{\S}$ Department of Medicine, Renal Division, Brigham and Women's Hospital and Harvard Medical School, Boston, Massachusetts 02115
}

\begin{abstract}
The effect of insulinopenic diabetes on the expression of glucose transporters in the small intestine was investigated. Enterocytes were sequentially isolated from jejunum and ileum of normal fed rats, streptozotocin-diabetic rats, and diabetic rats treated with insulin. Facilitative glucose transporter (GLUT) 2, GLUT5, and sodium-dependent glucose transporter 1 protein content was increased from 1.5- to 6-fold in enterocytes isolated from diabetic animals in both jejunum and ileum. Insulin was able to reverse the increase in transporter protein expression seen after induction of diabetes.

There was a four- to eightfold increase in the amount of enterocyte glucose transporter mRNA after diabetes with greater changes in sodium-dependent glucose transporter 1 and GLUT2 than in GLUT5 levels. In situ hybridization showed that after the induction of diabetes there was new hybridization in lower villus and crypt enterocytes that was reversed by insulin treatment.

Thus, the increase in total hexose transport caused by diabetes is due to a premature expression of hexose transporters by enterocytes along the crypt-villus axis, causing a cumulative increase in enterocyte transporter protein during maturation. These changes are likely to represent an adaptive response by the organism to increase nutrient absorption in a perceived state of tissue starvation. These adaptive changes may lead to exacerbation of hyperglycemia in uncontrolled diabetes. $(J$. Clin. Invest. 1994.93:578-585.) Key words: glucose transport • diabetes $\bullet$ intestine $\bullet$ fructose $\bullet$ gene regulation
\end{abstract}

\section{Introduction}

Small intestinal epithelial cells have a major role in the digestion and absorption of dietary carbohydrates, which begins with the hydrolysis of complex carbohydrates to monosaccharides. The latter are then absorbed by the epithelium by both energy-requiring and energy-independent mechanisms (1). One of these processes involves the sodium-dependent glucose

Address correspondence to Charles F. Burant, M.D., Ph.D., Department of Medicine, MC1027, 5841 S. Maryland Avenue, Chicago, IL 60637.

Received for publication 4 June 1993 and in revised form 12 August 1993

J. Clin. Invest.

(C) The American Society for Clinical Investigation, Inc. 0021-9738/94/02/0578/08 $\$ 2.00$

Volume 93, February 1994, 578-585 cotransporter (SGLT1) ${ }^{1}$ located at the brush border membrane (BBM) that initiates the uptake of glucose, a highly efficient process because of its coupling with the inwardly directed sodium gradient of the cell (2). Efflux of hexoses from the enterocyte occurs via a member of the facilitative glucose transporter gene family (GLUT2) which has been localized by immunohistochemistry to the basolateral membrane of enterocytes (3). GLUT2 has a low affinity for glucose (4), but under conditions of active glucose transfer across the BBM the kinetic properties of GLUT2 allow for the efficient, unidirectional movement of glucose out of the enterocyte into the interstitial space and blood stream. Fructose, another major dietary sugar, is transported by a carrier distinct from SGLT1 since mutations in SGLT1 do not affect fructose transport (5) and dietary manipulations that affect glucose flux do not affect fructose flux (6). Recently, we have presented evidence that GLUT5 is a high affinity fructose transporter of the $\operatorname{BBM}(7,8)$. Additionally, we (4) and others (9) have shown that GLUT2 has fructose transport activity and likely mediates the basolateral exit of fructose from the enterocyte.

Hexose transport by the small intestine is modulated during development and under different physiological states. For instance, the intestinal uptake of both glucose and fructose changes with the developmental age of the rat, independent of diet (10). In contrast, adult rats fed diets enriched in glucose show a specific upregulation of glucose transport activity in both the BBM (11) and the basolateral membrane (12). Recently, changes in the BBM transport activity were shown to be due in part to increased sodium-dependent glucose transporter expression in enterocytes emerging from the crypt regions of the small intestinal mucosa (13).

Insulinopenic diabetes is another condition that may alter intestinal glucose transport, as well as other digestive and nutritive processes. Besides hypertrophy of specific mucosal regions, there are alterations in hydrolases and water, electrolyte, and nutrient absorption $(14,15)$. Glucose absorption is increased in both the BBM and in the basolateral membranes of the enterocyte (15-17). Using the photolabel $\left[{ }^{3} \mathrm{H}\right]$ phlorizin, which preferentially labels the sodium-dependent glucose transporter, Fedorak et al. (16) showed a significant increase in the number of these transporters in the BBM of lower villus and crypt enterocytes. This increase paralleled the increase in transport activity which was increased in the crypt regions to a greater extent than the villus tip regions. Insulinopenic diabetes also increases basolateral membrane glucose transport as determined by uptake and cytochalasin B binding studies, the latter

1. Abbreviations used in this paper: BBM, brush border membrane; GAPDH, glyceraldehyde 3-phosphate dehydrogenase; GLUT, facilitative glucose transporter; SGLT, sodium-dependent glucose transporter. 
a reagent that specifically labels facilitative glucose transporters (17). A recent report using human glucose transporter cDNA probes has shown increased levels of GLUT2 and SGLT1 mRNA by RNA blotting mucosal scrapings of chronically diabetic rats (18).

We have recently cloned the rat homologues of GLUT5 (8) and SGLT1 (Lee, W.-S., Y. Kanai, R. G. Wells, and M. Hediger, manuscript submitted for publication) which have allowed the development of antisera specific for these transporters. In this report, we use the specific rat $\mathrm{cDNAs}$ and antisera to investigate the region-specific responses and the molecular mechanisms responsible for the increase in glucose transport after the induction of insulinopenic diabetes.

\section{Methods}

Animals. Male Sprague-Dawley rats (Charles River Breeding Laboratories, Inc., Wilmington, MA) weighing 200-220 g were used in all experiments. They were kept in a 12-h light/dark cycle and were fed a standard chow diet ad lib. except for a 12 -h fast before injection of streptozotocin. The protocol for the studies was approved by the Animal Resource Center at the University of Chicago. Diabetes was induced by intravenous injection of $50 \mathrm{mg} / \mathrm{kg}$ of streptozotocin. Blood glucose was checked daily, and only those animals whose readings were $>300$ $\mathrm{mg} / \mathrm{dl}$ were considered diabetic. Some streptozotocin-diabetic rats were treated with insulin (Novolin 70/30; Novo Nordisk, Copenhagen, Denmark) $6 \mathrm{wk}$ after the induction of diabetes by subcutaneous injection of insulin at $\sim 20 \mathrm{U} / \mathrm{kg}$ twice daily for $1 \mathrm{wk}$. This regimen nearly normalized blood glucose and allowed the diabetic rats to regain weight at a rate similar to controls. The rats were killed $45 \mathrm{~d}$ after the induction of diabetes or after $1 \mathrm{wk}$ of insulin injections. Characteristics of the experimental animals are presented in Table I.

Tissue preparation. Rats were anesthetized by intraperitoneal injection of chloral hydrate $(700 \mathrm{mg} / \mathrm{kg})$. The small intestine was removed from Treitz's ligament to the cecum and was divided into thirds. The proximal one-third was designated jejunum, and the distal one-third was designated ileum as described previously (19). A 5-10-mm section of each segment was fixed for in situ hybridization, and the rest of the intestine was used for isolation of enterocytes.

Enterocyte fractionation of jejunum and ileum. A modification of the procedure of Weiser (20) was used. Briefly, intestinal segments were filled with $20 \mathrm{mM}$ EDTA, $1 \mathrm{mM}$ dithiothreitol, and $0.05 \%$ ( $\mathrm{vol} /$ vol) mercaptoethanol in PBS (15.2 $\mathrm{mM} \mathrm{Na}_{2} \mathrm{HPO}_{4}, 4.4 \mathrm{mM} \mathrm{KH}_{2} \mathrm{PO}_{4}$, and $140 \mathrm{mM} \mathrm{NaCl}, \mathrm{pH} 7.4$ ) and were incubated at $37^{\circ} \mathrm{C}$ in PBS with 20 $\mathrm{mM}$ EDTA. Fractions were isolated after sequential 7-min incubation periods, except for the final fraction which was incubated for $10 \mathrm{~min}$ followed by gentle massage to ensure complete removal of crypt cells. At the end of each period, loops were emptied and flushed to obtain

Table I. Clinical Parameters of Control and Diabetic Rats

\begin{tabular}{lcccc}
\hline & $\begin{array}{c}\text { Weight } \\
\text { start }\end{array}$ & $\begin{array}{c}\text { Weight } \\
\text { end }\end{array}$ & $\begin{array}{c}\text { Blood } \\
\text { glucose }\end{array}$ & Glycohemoglobin \\
\hline & $g$ & $g$ & $m g / d l$ & $\%$ \\
Control & $272 \pm 8$ & $386 \pm 11$ & $121 \pm 7$ & $6.1 \pm 0.9$ \\
Diabetes & $278 \pm 10$ & $235 \pm 15^{*}$ & $>400^{*}$ & $13.8 \pm 2.9^{*}$ \\
Insulin-treated & $268 \pm 15$ & $271 \pm 7^{*}$ & $169 \pm 21^{\ddagger}$ & $\mathrm{ND}$ \\
\hline
\end{tabular}

Diabetes was induced by administration of streptozotocin $(50 \mathrm{mg} / \mathrm{kg}$ body wt), and tissues were harvested after $45 \mathrm{~d}$ or after an additional week of insulin treatment. Glucose concentration and glycohemoglobin levels at the time of tissue harvest were determined by blood obtained from cardiac puncture. Values shown are mean \pm SE for 4-10 animals. ND, not determined. ${ }^{*} P \leq 0.05$ vs control. ${ }^{\ddagger} P \leq 0.05$ vs control and diabetic. each fraction. Isolated cells were centrifuged at $1,500 \mathrm{~g}$ for $2 \mathrm{~min}$ and were resuspended in PBS with protease inhibitors $(10 \mu \mathrm{g} / \mathrm{ml}$ leupeptin, and $0.01 \%$ soybean trypsin inhibitor). Aliquots were processed for enzyme assays or RNA extraction, as described below. As described in the past (19), reciprocal gradients of sucrase activities were obtained in villus-to-crypt enterocyte fractions performed as described previously (21) and corresponded to sucrose and $\left[{ }^{3} \mathrm{H}\right]$ thymidine incorporation gradients previously reported (19) (results not shown).

RNA isolation. RNA was prepared immediately from $75 \%$ of the cells isolated from each enterocyte fraction by homogenization in $7 \mathrm{M}$ guanidium thiocyanate, $0.5 \% N$-lauryl sarcosine, and $25 \mathrm{mM}$ sodium citrate, $\mathrm{pH}$ 7.0. The RNA was isolated by centrifugation through a cushion of $\mathrm{CsCl}(21)$. Poly (A) ${ }^{+}$mRNA was prepared by oligo dT chromatography and was quantitated by reading absorbance at 260 and $280 \mathrm{~nm}$.

Northern blotting. 4-5 $\mu \mathrm{g}$ of poly (A $)^{+}$mRNA from each enterocyte fraction was subjected to $1 \%$ agarose $/ 6 \%$ formaldehyde electrophoresis and then was transferred to a nitrocellulose membrane by capillary action. The blots were incubated with ${ }^{32} \mathrm{P}$-labeled-cDNA probes corresponding to rat SGLT1 (Hediger, M. et al., unpublished results), rat GLUT5 (8), or an 1,800-bp rat GLUT2 EcoRI fragment isolated from a rat liver cDNA library prepared as previously described (8). The membranes were washed in $0.1 \times$ SSC, $0.1 \%$ SDS for $30 \mathrm{~min}$ at $25^{\circ} \mathrm{C}$ and then for $30 \mathrm{~min}$ at $56^{\circ} \mathrm{C}$ and were autoradiographed for varying times for subsequent quantitation. Each blot was additionally probed with a glyceraldehyde 3-phosphate dehydrogenase (GAPDH) cDNA (American Type Culture Collection, Rockville, MD), a constitutively expressed transcript, to normalize for loading of the gels (see Results).

Total membrane isolation. One-quarter of each enterocyte fraction was homogenized in $5 \mathrm{ml} 10 \mathrm{mM}$ Tris, pH 7.4, $250 \mathrm{mM}$ sucrose, $1 \mathrm{mM}$ EDTA, $1 \mathrm{mM}$ PMSF, and $1 \mu \mathrm{g} / \mathrm{ml}$ each leupeptin and pepstatin, and was centrifuged at $900 \mathrm{~g}$ for $10 \mathrm{~min}$. The resulting pellet was rehomogenized and recentrifuged as above. The supernates were combined and centrifuged at $100,000 \mathrm{~g}$ for $75 \mathrm{~min}$. The pellet was solubilized in $100-200 \mu$ l of the above buffer containing $0.5 \%$ deoxycholate and $0.5 \%$ Triton X-100.

Western blotting. $100 \mu \mathrm{g}$ of membrane protein was subjected to SDS-PAGE using a $10 \%$ resolving gel, and the proteins were electrophoretically transferred to Immobilon-P membranes (Millipore Corp., Bedford, MA). The filters were blocked with 5\% Carnation Instant Milk $^{\circledR}$ in Tris-buffered saline ( $20 \mathrm{mM}$ Tris, $\mathrm{pH} 7.4,50 \mathrm{mM} \mathrm{NaCl}$, and $0.05 \%$ Tween 20 ) and were probed with antisera specific for the glucose transporter of interest. After washing three times in $50 \mathrm{ml}$ Tris-buffered saline, the blots were developed by the addition of ${ }^{125}$ I-labeled protein A (Amersham Corp., Arlington Heights, IL) and were autoradiographed for various periods of time to attain images within the linear range of the autoradiographic film (Kodak XAR; Eastman Kodak Co., Rochester, NY) before subsequent quantitation. Rat GLUT2 antisera was obtained from East Acres Biologicals (Southbridge, MA), lot 160708. ratGLUT5 antisera was produced by injection of synthetic peptides corresponding to the last 17 amino acids of the predicted rat GLUT5 sequence, EKEEKELNDLPPATREE (8), into rabbits after emulsification in Freund's adjuvant and was a gift of Dr. Sam Cushman (National Institutes of Health, Bethesda, MD). The rat SGLT1 antibodies were raised against a peptide, KPIPDVHLYRLCWSLRNST, that corresponds to amino acids 550-567 of the predicted protein sequence of rat SGLT1 ( Lee, W.-S., Y. Kanai, R. G. Wells, and M. Hediger, manuscript submitted for publication ) and were prepared by a commercial source (Research Genetics, Huntsville, AL). The specificity of the latter two antisera was determined by the ability of the immunizing peptide to eliminate signal on Western blots and by the ability of the antisera to specifically immunoprecipitate rat GLUT5 and rat SGLT1 protein synthesized using transporter mRNA in a rabbit reticulocyte lysate (data not shown).

Quantitation of glucose transporter mRNA and protein content. The autoradiographs were scanned with a Scan Jet IIc (Hewlett-Packard Co., Palo Alto, CA) at 400 dots per inch resolution and the images were analyzed (Image 1.42 program; National Institutes of Health) using a 
256 gray scale image. RNA and protein samples from control and diabetic animals, as well as insulin-treated animals where appropriate, were simultaneously electrophoresed and probed with labeled cDNA or antisera on the same blot.

In situ hybridization. In situ hybridization was performed as described previously (8). The synthetic RNA was prepared from a 486bp EcoRI fragment of GLUT5, a 718-bp EcoRI/HindIII fragment of GLUT2, or a 550-bp BamHI fragment of SGLT1 after subcloning into pGEM4Z and was linearized with an appropriate restriction enzyme. The subsequent sense or antisense synthetic RNAs were transcribed using SP6 or T7 RNA polymerase in the presence of $\alpha^{35}$ S-UTP (8). Samples from control, diabetic, and insulin-treated rats were mounted on the same slide and were probed simultaneously.

Statistical analysis. Differences between groups were determined by Student's $t$ test with significance taken as $P \leqslant 0.05$.

\section{Results}

Clinical features of rats. Rats were treated with streptozotocin and were maintained for $6 \mathrm{wk}$ in a protocol we have used previously to study the effect of chronic diabetes on intestinal function (19). Progressive weight loss was seen in the rats treated with streptozotocin (Table I). This was accompanied by hyperglycemia and elevated glycosylated hemoglobin levels. In previous studies from this laboratory, streptozotocin-diabetic rats prepared under the same protocol had insulin levels that were below the limit of detection. Diabetic rats that were treated with insulin nearly normalized their blood glucose levels ( Table I) and initiated weight gain at a rate comparable with control (not shown).

Expression of glucose transporters in fractionated enterocytes. Incubation of small intestinal segments in an EDTAcontaining buffer results in the progressive removal of enterocytes from villus to crypts. Gradients of sucrase-isomaltase and thymidine incorporation are obtained using this method (19), but because of admixing of fractions it provides a qualitative difference in the expression of macromolecules along the cryptvillus axis. The amount of glucose transporter protein expressed in the enterocytes was quantitated by SDS-PAGE. Identical amounts of total, post-nuclear membrane protein from enterocyte fractions from jejunum and ileum were subjected to Western blot analysis using specific anti-glucose transporter antibodies. The GLUT2 antisera has been previously characterized and recognizes a band of 60-62 kD. The rat GLUT5 and rat SGLT1 antisera recognize proteins of 57-60 and 70-72 kD, respectively (Fig. 1, insets). Detection of these bands by both antisera can be eliminated by preincubation with the immunizing peptide. For each of the glucose transporters, there was more immunoreactive protein isolated from jejunum compared with ileum (Fig. 1, insets). Enterocyte fractionation from control intestine showed a slightly greater amount of GLUT2 and GLUT5 protein in the mid and upper villus fractions as compared with crypt fractions, but these changes were not significantly different. SGLT1 protein expression appeared more evenly distributed along the villuscrypt axis (Fig. $1 C$ ).

Glucose transporter protein levels are increased in diabetic rats and are reversed by insulin treatment. Hypertrophy of the gastrointestinal tract in diabetic rats is accompanied by a 40 and $10 \%$ increase in total enterocyte protein isolated from diabetic jejunum and ileum, respectively. However, there is no difference in the amount of protein recovered per DNA con- tent of the enterocyte fractions (17, and Chang, E. B., manuscript in preparation). Although total post-nuclear membrane protein recovered was increased in the diabetic samples, there was no change in the total membrane protein recovered per milligram of homogenate protein (Table II). Insulin treatment of diabetic animals likewise had no effect on the recovery of membrane protein (Table II).

Streptozotocin-induced diabetes resulted in a significant increase in the amount of GLUT2 protein expressed in both jejunal and ileal enterocytes (Fig. $1 A$ ). The largest relative increase was seen in the fractions derived from the lower villus and crypt in both the jejunum and ileum with up to sixfold more protein expressed in these fractions. Because of the lower basal levels of GLUT2 expression in the ileum, the fold increase was slightly greater in magnitude in the ileum than in the jejunum (Fig. 1, $A$ vs $B$ ), but the absolute amount of GLUT2 protein was still less in ileal as compared with jejunal enterocytes (Fig. $1, A$ and $B$, insets).

The expression of GLUT5 protein in the same enterocyte fractions was also increased by diabetes (Fig. 1, $C$ and $D$ ). Again, the increase was more marked in the samples from the crypt as opposed to the villus tip, and, as with GLUT2, there was on average a greater amount of GLUT5 in the jejunum than in the ileum with up to a fourfold increase in GLUT5 protein in the crypt fractions (Fig. 1, $C$ and $D$ ). The change in expression of SGLT1 in these samples was more variable after the induction of diabetes, with a much greater change seen in jejunum as compared with ileum. However, there was a signifcant increase in the content of SGLT1 protein in the ileal crypt fraction as well as in all jejunal fractions.

Insulin treatment of the rats markedly improved blood glucose levels (Table II) and resulted in less total protein from each enterocyte fraction as compared with control (not shown). A reduction in the amount of immunoreactive GLUT2, GLUT5, and SGLT1 to near control levels was seen per milligram of post-nuclear membrane protein in the jejunal and ileal fractions from the insulin-treated diabetic rats (Fig. 2).

Glucose transporter $m R N A$ is increased in diabetes. To examine the mechanism responsible for increased glucose transporter protein expression in diabetic rats, total cellular RNA was prepared from the enterocyte fractions and was enriched for poly (A) ${ }^{+}$mRNA by oligo dT chromatography. In these experiments, six fractions of enterocytes were isolated. The amount of total RNA recovered per cell protein was unchanged after the induction of diabetes (Table II). To normalize for slight differences in the poly $(\mathrm{A})^{+} \mathrm{mRNA}$ loaded in each lane, each Northern blot was probed with ${ }^{32} \mathrm{P}$-labeled GAPDH cDNA. Although GAPDH levels rose approximately twofold per microgram of poly $(A)^{+}$RNA after the induction of diabetes, similar amounts were expressed in each of the enterocyte fractions from jejunum and ileum per microgram of RNA loaded on the gel (Fig. 3). The data for the subsequent Northern blots were normalized within each fractionation experiment to GAPDH mRNA levels, that is, the amount of glucose transporter mRNA detected was first divided by the amount of GAPDH detected in the same sample. The calculated amount in each diabetic sample was then divided by the calculated amount in the control sample within each of the crypt-to-villus fractions to arrive at the relative amount of diabetic/control mRNA. Although this correction will decrease relative changes in the diabetic samples as compared with control, it increases 
GLUT2

A

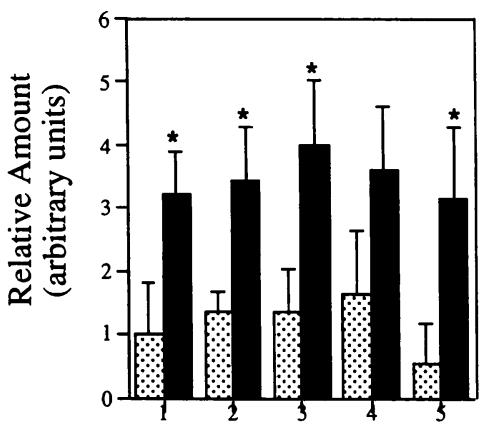

Fraction Number (Tip $\rightarrow$ Crypt)

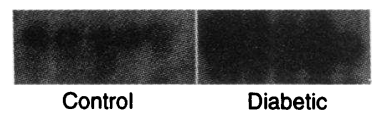

B

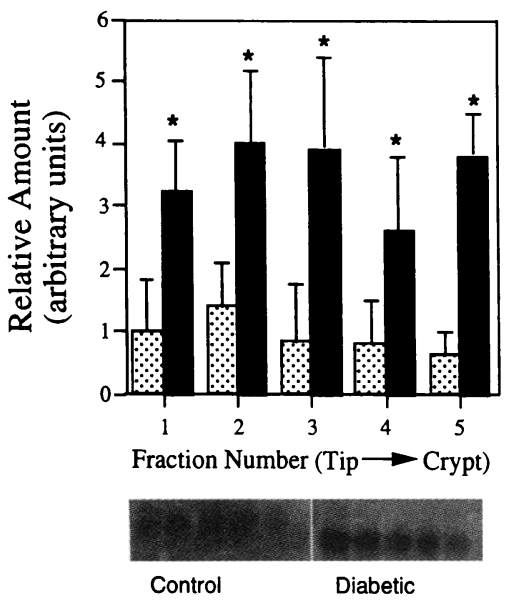

GLUT5

C
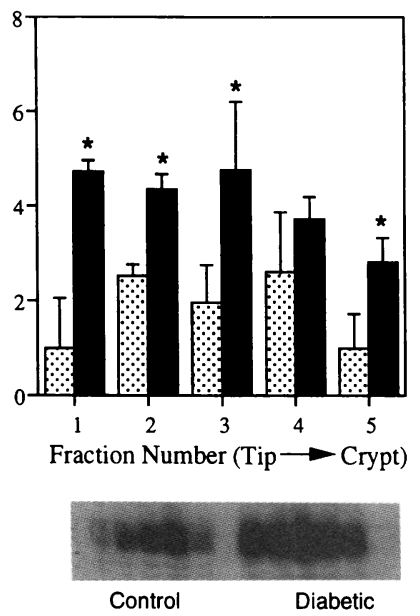

D

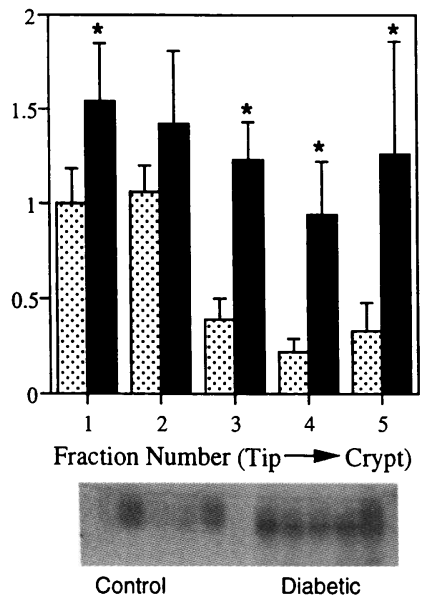

SGLT1

E

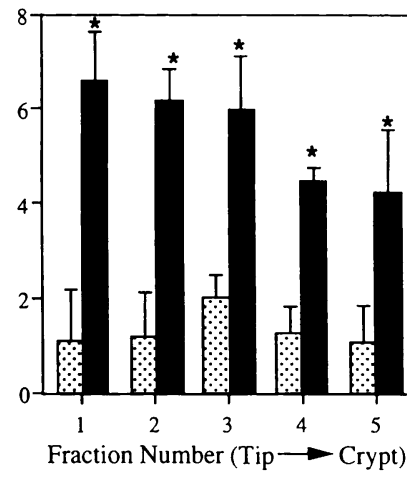

$\frac{5}{5}$

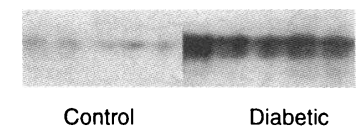

F.

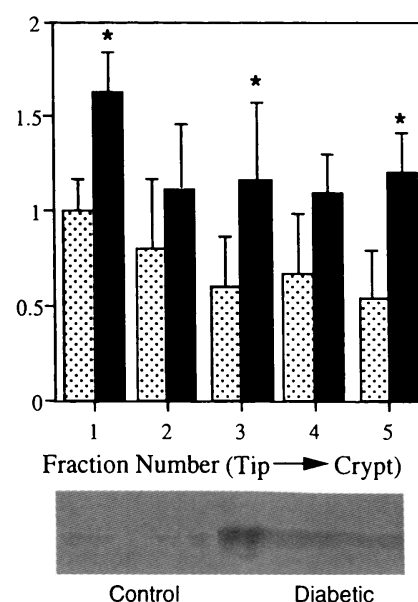

Figure 1. Effect of diabetes on glucose transporter protein expression in enterocytes. Enterocytes were sequentially isolated from villus tip to crypt, and $100 \mu \mathrm{g}$ of total post-nuclear membrane protein was isolated and separated by SDS-PAGE as described in Methods. Relative amount of immunoreactive protein, GLUT2 $(A$ and $B)$, GLUT5 $(C$ and $D)$, and SGLT1 ( $E$ and $F)$ from jejunum $(A, C$, and $E)$ or ileum $(B, D$, and $F$ ) in enterocyte fractions from control $\square$ and diabetic $\square$ rats. Each bar represents the mean \pm SE from three to four determinations from individual animals. *Significant differences between control and diabetic samples at $P \leq 0.05$. (Insets) Representative autoradiograph of GLUT2, GLUT5, or SGLT1 protein from the fractionated small intestinal segments isolated from control and diabetic animals.

the accuracy of quantitation. To eliminate intraassay variability, control- and diabetic-derived RNA preparations from each set of rats in an experiment were chromatographed on the same gel, were transferred to the same piece of nitrocellulose, and were probed sequentially for each transporter as well as for GAPDH mRNA.

Table II. Effect of Diabetes and Insulin Treatment on Protein and RNA Levels

\begin{tabular}{|c|c|c|c|c|c|c|}
\hline & \multicolumn{2}{|c|}{ Total membrane protein } & \multicolumn{2}{|c|}{ BBM protein } & \multicolumn{2}{|c|}{ RNA } \\
\hline & \multicolumn{2}{|c|}{ Homogenate protein } & \multicolumn{2}{|c|}{ Total membrane protein } & \multicolumn{2}{|c|}{ Homogenate protein } \\
\hline & Jejunum & Ileum & Jejunum & lleum & Jejunum & Ileum \\
\hline & \multicolumn{2}{|c|}{$\mu g$} & \multicolumn{2}{|c|}{$\mu g$} & \multicolumn{2}{|c|}{$\mu g$} \\
\hline Control & $0.19 \pm 0.05$ & $0.21 \pm 0.11$ & $0.076 \pm 0.012$ & ND & $17.6 \pm 5.9$ & $21.1 \pm 12.8$ \\
\hline Diabetes & $0.22 \pm 0.09$ & $0.16 \pm 0.08$ & $0.083 \pm 0.009$ & ND & $13.4 \pm 4.3$ & $16.9 \pm 7.2$ \\
\hline Insulin-treated diabetic & $0.21 \pm 0.04$ & $0.16 \pm 0.11$ & $0.078 \pm 0.21$ & ND & ND & ND \\
\hline
\end{tabular}

Total cellular membranes, BBM, and total cellular RNA were isolated from jejunal and ileal enterocytes as described in Methods. The values shown are the mean $\pm \mathrm{SE}$ of the enterocyte fractions from three to four individual animals in each group. ND, not determined. 

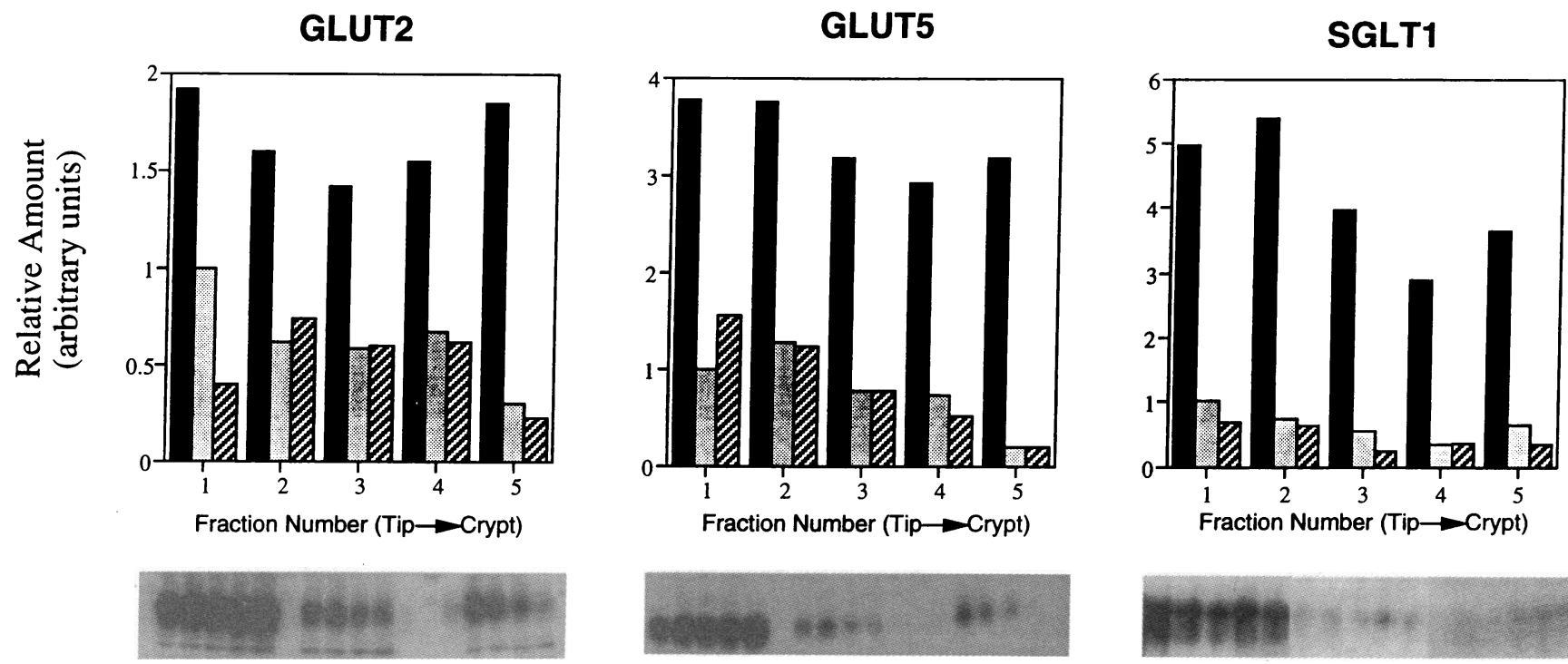

Figure 2. Effect of insulin on glucose transporter expression in diabetic rat jejunum. After $45 \mathrm{~d}$ of diabetes, rats were treated for 1 wk with twice daily injections of insulin, resulting in near normalization of blood glucose. Relative amounts of immunoreactive protein of the enterocyte fractions from control $\mathbf{a}$, diabetic $\mathbf{a}$, and insulin-treated diabetic $\square$ rats. Shown is the mean of two experiments. (Insets) Representative autoradiograph of GLUT2, GLUT5, or SGLT1 protein from the fractionated small intestinal segments isolated from control, diabetic, and insulintreated diabetic animals.

Only a single transcript for each transporter was detected in RNA from both control and diabetic intestine. When Northern blots were probed with ${ }^{32} \mathrm{P}$-labeled cDNAs, the relative size of the GLUT2, GLUT5, and SGLT1 transcripts were 2.8, 3.2, and $4.3 \mathrm{~kb}$, respectively. As with the glucose transporter proteins, in control animals there was more GLUT2 mRNA in jejunal- than in ileal-derived enterocytes (Fig. 3). After induction of diabetes, there was a marked increase in the amount of these transporter mRNAs, with a greater relative increase in the enterocytes from the crypt compared with the villus cells (Figs. 3 and 4). The increase in mRNA was approximately twofold for each transporter in the fractions from the villus tip, with the exception of GLUT5 in jejunum which increased to four- to eightfold in the crypt fractions. The increases in mRNA for the most part paralleled the increases seen in protein expression. There were increases in both jejanum and ileum; the changes were greater for GLUT2 and SGLT1 and smaller for GLUT5, and the increases in glucose transporter expression were seen in both jejunum and ileum. The largest increases were in GLUT2 and SGLT1 mRNA in both jejunum and ileum with a much smaller rise in the relative amount of GLUT5 mRNA (Figs. 3 and 4).

In situ hybridization. To confirm the observed increase and altered distribution of transporter mRNA expression after the induction of diabetes, we performed in situ hybridization studies on jejunal sections from control, diabetic, and insulintreated diabetic animals. Sections from each experimental group were mounted on the same slide, and hybridization, washing, and development were performed together. As shown previously for GLUT5 (8), the expression of both GLUT2 and GLUT5 mRNAs begins to appear in enterocytes emerging from the crypts, is highest in the midvillus regions, and decreases at the villus tip (Fig. 5). By contrast, SGLT1 is more evenly distributed in the bottom two-thirds of the villus with decreasing amounts in the villus tip. There was negligible signal within the crypts for any probe.

Although not quantitative, in the section from the diabetic animals there appeared to be an increase in GLUT2, GLUT5, and SGLT1 hybridization signal with a consistent pattern of earlier expression in the crypt-villus axis. This was particularly

Jejunum

\section{Control Diabetic}

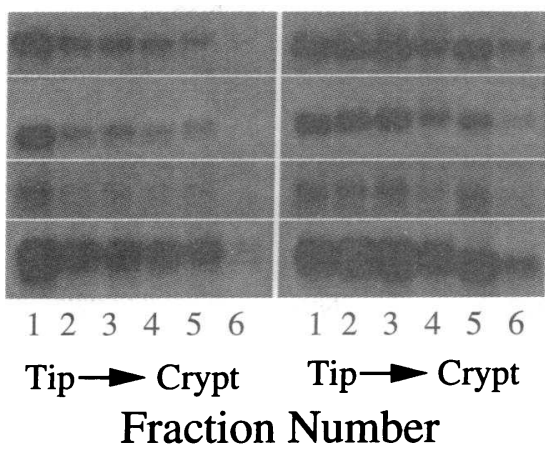

Ileum

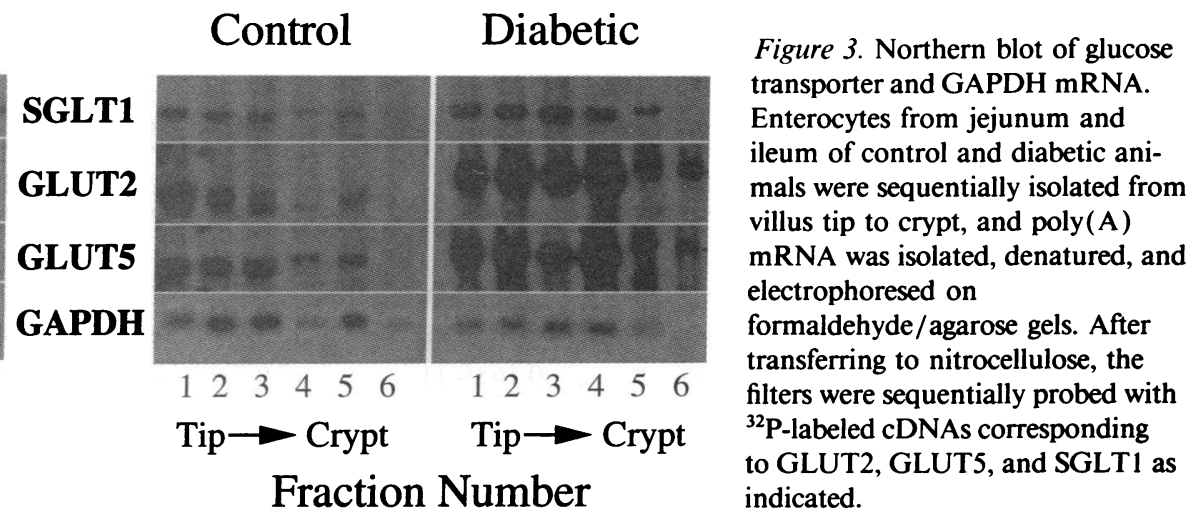


GLUT2
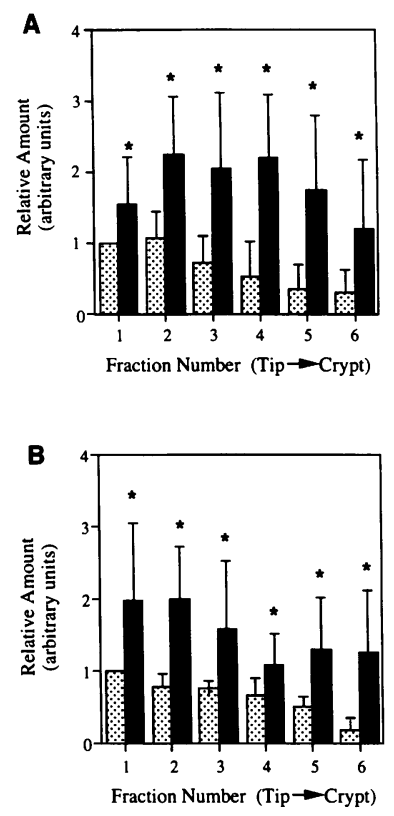

GLUT5

C

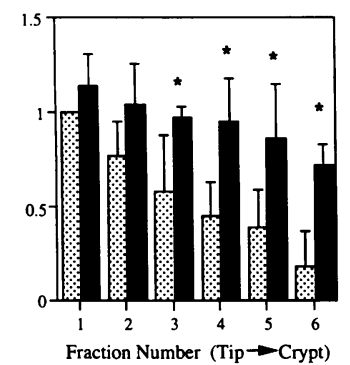

D

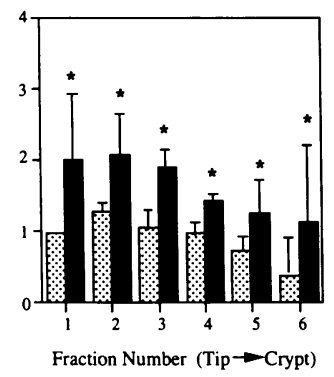

evident in GLUT2 and GLUT5 hybridizations (Fig. 5, $B, E$, and $H$ ). The insulin-treated group tended to show an apparent decrease in hybridization signal as compared with the diabetic sections with each probe and an elimination of signal in the lower villus and crypt regions seen in the diabetic jejunal sections (Fig. 5, $C, F$, and $I$ ).

\section{Discussion}

Diabetes is associated with hypertrophy of the small intestinal villi of the proximal small intestine, which results in a greater surface area for nutrient absorption in this state of relative tissue starvation (21). The increase in surface area cannot entirely account for the observed increases in glucose flux in the small intestine of diabetic rats. There is a compensatory increase in the amount of glucose flux on a per cell basis which may account for $50-70 \%$ of the increase in glucose transport $(16,17)$. Studies of phloridzin binding suggested that part of the increase is because of increased expression of sodium-dependent glucose transporters in the crypt fractions of the villus (16). The experiments described here show that there is indeed increased expression of SGLT1 protein in the cells isolated from the crypt of both the jejunum and ileum.

In addition to increased glucose transport activity in the BBM of diabetic animals, changes in glucose transport in the basolateral membrane of both jejunal (17) and ileal enterocytes (22) have been noted. The increase in activity was greatest in those enterocytes obtained from the lower villus and crypt. In addition, after the induction of diabetes, there is an increase in cytochalasin B binding, an antimetabolite that specifically inhibits facilitative glucose transport of the basolateral membrane $(17,22)$. GLUT2 is the only facilitative glucose transporter expressed in enterocytes (GLUT5 being a fructose transporter), and there is a marked increase in the amount of GLUT2 protein expressed in all fractions of enterocytes in both jejunum and ileum after the induction of diabetes, with the largest increase being in enterocytes isolated from the crypt. Thus, the increase in glucose flux across the enterocyte in strep-

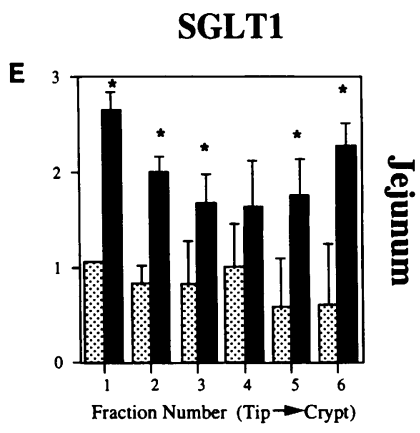

Figure 4. Effect of diabetes on the expression of glucose transporter mRNA. Enterocytes from jejunum $(A, C$, and $E)$ and ileum $(B, D$, and $E)$ from control $\square$ and diabetic $a$ rats were sequentially isolated from villus tip to crypt as described in Fig. 3 . The resultant autoradiograms were quantitated by scanning densitometry, and the relative amount of transporter was determined after normalization of each mRNA to GAPDH mRNA within each experiment (see Methods and Results). $A$ and $B$, GLUT2; $C$ and $D$, GLUT5; $E$ and $F$, SGLT1. Shown is the mean \pm SE. $n=3-4$ for each point. *Significant increase in diabetic above control mRNA content at $P \leq 0.05$. tozotocin-induced diabetes is due to increases in SGLT1 expression at the BBM and GLUT2 expression at the basolateral membrane.

Recently, we have shown that GLUT5 is a fructose transporter and have proposed that it is the primary fructose carrier of the $\operatorname{BBM}(5,8)$. Little is known about the changes in fructose transport in experimental or clinical diabetes, but the results presented here would suggest that, like glucose, the uptake of fructose would be increased in insulinopenic diabetes.

The increase in both sodium-dependent and facilitative glucose and fructose transporter protein levels seen after the induction of streptozotocin diabetes is apparently due to increased levels of mRNA in enterocytes in the villus-crypt axis that normally express these transporters. In addition, diabetes appears to change the pattern of expression of these genes along the villus-crypt axis, with the expression of these genes beginning to appear in relatively more immature cells of the lower villus and crypt. These changes in expression were noted by both RNA blotting of enterocyte fractions as well as by in situ hybridization.

The increases in glucose transporter expression with diabetes can be reversed by insulin treatment of the diabetic animals (Figs. 2 and 5). Thus, the observed changes are not due to a secondary effect of the streptozotocin. The mechanism by which insulinopenic diabetes increases transporter expression is not clear. Preliminary studies suggest that it is not simply lack of insulin causing the change, since glucose infusion and high carbohydrate feeding can increase facilitative glucose transporter expression (Burant, C. F., manuscript in preparation). This would suggest that increased glucose, either in the blood or intestine (secondary to hyperphagia associated with diabetes), may increase in the transporter levels. Similarly, high carbohydrate diets increase the expression of sodium-dependent glucose transporters in the small intestine of mice with the predominant increase in crypt enterocytes (11). In addition, it has been shown that both high carbohydrate diets and glucose infusion can increase the transport activity in the basolateral membrane in rat enterocytes (12). The mechanism by 

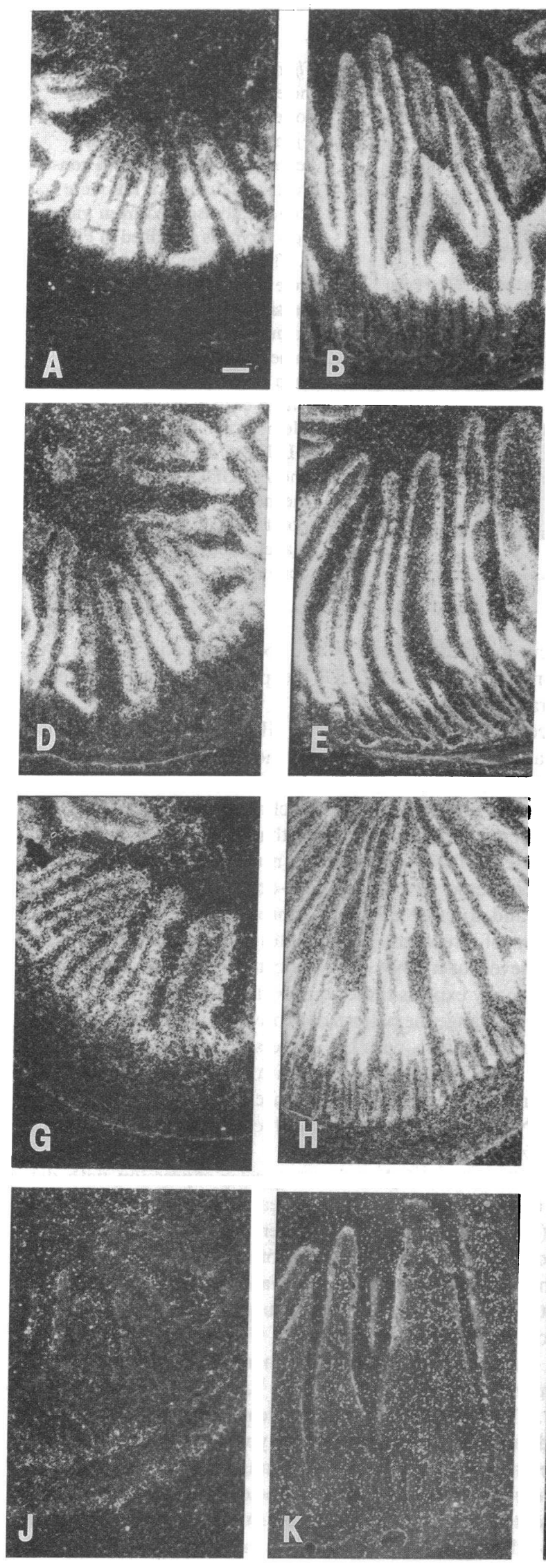
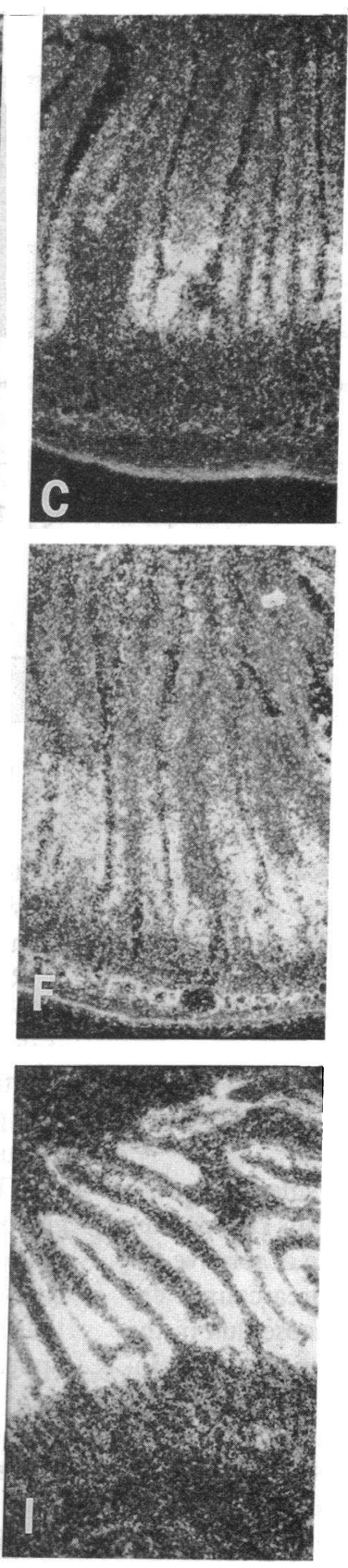

Figure 5. In situ hybridization of glucose transporters in jejunum from control, diabetic, and diabetic rats treated with insulin. Representative dark-field photomicrographs of jejunal sections hybridized with ${ }^{35} \mathrm{~S}$-labeled cRNA and developed $8 \mathrm{~d}$ later as described previously (8). Sections of jejunum from control $(A, D, G$, and $J)$, diabetic ( $B, E, H$, and $K)$, and insulin-treated diabetic rats $(C, F, I$, and $L$ ) hybridized with antisense SGLT1 $(A$, $B$, and $C)$, GLUT2 $(D, E$, and $F)$, GLUT5 ( $G$, $H$, and $I$ ) or a representative control, and sense $\operatorname{GLUT} 2(J, K$, and $L) \mathrm{cRNA}$. Bar $=20 \mu \mathrm{M}$. 
which increased loads of glucose to the enterocyte increases expression of glucose transporter proteins is not clear.

The increase in facilitative glucose transporter levels in the small intestine in response to insulinopenic diabetes is contrary to reported observations in muscle and adipose tissue. A decrease in the insulin-responsive glucose transporter (GLUT4) mRNA and/or protein has been observed after the induction of diabetes $(23,24)$. In contrast, the levels of rat liver GLUT2 mRNA and protein levels have either been reported to rise (25), have no change (26), or have a biphasic response to streptozotocin-induced diabetes (27). The mechanism for tissue differences in controlling transporter expression is not yet understood.

In conclusion, we show at the molecular and cellular levels that diabetes causes an increase in the levels of glucose (SGLT1 and GLUT2) and fructose (GLUT5) transporters in the small intestine. This is due, at least in part, to mechanisms that include induction of precocious expression of these genes in crypt cells and increased mRNA and protein abundance by each enterocyte. These increases in transporter expression may represent an adaptive mechanism by the organism to increase nutrient uptake in a time of relative tissue starvation. The increase in absorptive capacity of the small intestine for carbohydrate along with hyperphagia associated with uncontrolled diabetes may lead to an exacerbation of the hyperglycemia seen in this condition.

\section{Acknowledgments}

The authors thank Dr. Sam Cushman for the gift of GLUT5 antisera, and Dr. Graeme Bell for his helpful comments and suggestions throughout these studies.

These studies were supported by National Institutes of Health grants DK-38510, DK-42086, and DK-43632, the Marilyn Simpson Trust Fund, and the Juvenile Diabetes Foundation International. C. F. Burant is a recipient of a Career Development Award from the American Diabetes Association.

\section{References}

1. Crane, R. K. 1975. The intestinal absorption of sugars. In Physiological Effects of Food Carbohydrates. J. A. Hodges, editor. American Chemical Society, Washington DC. 2-19.

2. Hediger, M. A., M. J. Coady, T. S. Ikeda, and E. M. Wright. 1987. Expression cloning and cDNA sequencing of the $\mathrm{Na}^{+}$/glucose co-transporter. Nature (Lond.). 330:379-381.

3. Thorens, B., Z.-Q. Cheng, D. Brown, and H. F. Lodish. 1990. Liver glucose transporter: a basolateral protein in hepatocytes and intestine and kidney cells. Am. J. Physiol. 259 (Cell Physiol. 28): C279-C285.

4. Burant, C. F., and G. I. Bell. 1992. Facilitative glucose transporters: evidence for similar substrate binding sites in functionally monomeric proteins. Biochemistry. 31:10414-10420.
5. Wright, E. M., E. Turk, B. Zabel, S. Mundlos, and J. Dyer. 1991. Molecular genetics of intestinal glucose transport. J. Clin. Invest. 88:1435-1440.

6. Solberg, D. H., and J. M. Diamond. 1987. Comparison of different dietary sugars as inducers of intestinal sugar transporters. Am. J. Physiol. 252 (Gastrointest. Liver Physiol. 15): G574-G584.

7. Burant, C. F., J. Takeda, E. Brot-Laroche, G. I. Bell, and N. O. Davidson 1992. GLUT5 is the fructose transporter of the small intestine and sperm. J. Biol Chem. 265:13276-13282.

8. Rand, E. B., A. M. DePaoli, G. I. Bell, and C. F. Burant. 1993. Cloning, sequence and functional expression of the rat fructose transporter, GLUT5. Am. J. Physiol. 262(Gastrointest. Liver Physiol. 25): G1169-G1176.

9. Colville, C. A., M. J. Seatter, T. J. Jess, G. W. Gould, and H. M. Thomas. 1993. Kinetic analysis of the liver-type (GLUT2) and brain-type (GLUT3) glucose transporters in Xenopus oocytes: substrate specificities and effects of transport inhibitors. Biochem. J. 290:701-706.

10. Toloza, E. M., and J. Diamond. 1992. Ontogenetic development of nutrient transporters in rat intestine. Am. J. Physiol. 263 (Gastrointest. Liver Physiol. 26): G593-G604.

11. Ferraris, R. P., S. A. Villenas, B. A. Hirayama, and J. Diamond. 1992. Effect of diet on glucose transporter site density along the intestinal crypt-villus axis. Am. J. Physiol. 262 (Gastrointest. Liver Physiol. 25): G1060-G1068.

12. Cheesman, C. I., and D. D. Maenz. 1989. Rapid regulation of D-glucose transport in basolateral membrane of rat jejunum. Am. J. Physiol. 256 (Gastrointest. Liver Physiol. 19): G878-G883.

13. Ferraris, R. P., and J. Diamond. 1992. Crypt-villus site of glucose transporter induction by dietary carbohydrate in mouse intestine. Am. J. Physiol. 262 (Gastrointest. Liver Physiol. 25): G1069-G1073.

14. Miller, D. L., and H. P. Schedl. 1976. Effects of experimental diabetes on intestinal absorption in the rat. Proc. Soc. Exp. Biol. Med. 152:589-592.

15. Philpott, D. J., J. D. Butzner, and J. B. Meddings. 1992. Regulation of intestinal glucose transport. Can. J. Physiol. 70:1201-1207.

16. Fedorak, R. N., E. B. Chang, J. L. Madara, and M. Field. 1987. Intestinal adaptation to diabetes. J. Clin. Invest. 79:1571-1578.

17. Debnam, E. S., H. Y. Ebrahim, and D. J. Swaine. 1990. Diabetes mellitus and sugar transport across the brush-border and basolateral membranes of the rat jejunal enterocytes. J. Physiol. (Lond.). 424:13-25.

18. Miyamoto, K., K. Hase, Y. Taketani, H. Minami, T. Oka, Y. Nakabou, and $\mathrm{H}$. Hagihira. 1991. Diabetes and glucose transporter gene expression in rat small intestine. Biochem. Biophys. Res. Commun. 181:1110-1117.

19. Hoffman, L. R., and E. B. Chang. 1992. Altered regulation of regional sucrase-isomaltase expression in diabetic rat intestine. Am. J. Physiol. 262 (Gastrointest. Liver Physiol. 25): G982-G989.

20. Weiser, M. M. 1973. Intestinal epithelial cell surface membrane glycoprotein synthesis. J. Biol. Chem. 248:2536-2541.

21. Schedl, H. P., and H. D. Wilson. 1971. Effects of diabetes on intestinal growth in the rat. J. Exp. Zool. 176:487-496.

22. Debnam, E. S., and G. Chowrimootoo. 1992. Streptozotocin diabetes and sugar transport by rat ileal enterocytes: evidence for adaptation caused by an increased luminal nutrient load. Biochim. Biophys. Acta. 1107:86-92.

23. Kahn, B. B., M. J. Charron, H. F. Lodish, S. W. Cushman, and J. S. Fier. 1989. Differential regulation of two glucose transporters in adipose cells from diabetic and insulin-treated diabetic rats. J. Clin. Invest. 84:404-411.

24. Kahn, B. B., L. Rossetti, H. F. Lodish, and M. J. Charron. 1991. Decreased in vivo glucose uptake but normal expression of GLUT1 and GLUT4 in skeletal muscle of diabetic rats. J. Clin. Invest. 87:2197-2206.

25. Oka, Y., T. Asano, Y. Shibasake, J. L. Lin, K. Tsukuda, Y. Akanuma, and F. Takaku. 1990. Increased liver glucose-transporter protein and mRNA in streptozotocin-induced diabetic rats. Diabetes. 39:441-446.

26. Thorens, B., J. S. Flier, H. F. Lodish, and B. B. Kahn. 1990. Expression of two glucose transporters in rat liver is differentially regulated by fasting and refeeding and by diabetes and insulin treatment. Diabetes. 39:712-719.

27. Burcelin, R., M. Eddouks, J. Kande, R. Assan, and J. Girard. 1992. Evidence that GLUT-2 mRNA and protein concentrations are decreased by hyperinsulinemia and increased by hyperglycemia in liver of diabetic rats. Biochem. J. 288:675-679. 